\title{
Multiple Targets Tracking Using Kinematics in Wireless Sensor Networks
}

\author{
Akond Ashfaque Ur Rahman ${ }^{1}$, Atiqul Islam Mollah', Mahmuda Naznin ${ }^{3}$ \\ ${ }^{1}$ Software Engineer-Dohatec New Media, Dhaka, Bangladesh \\ ${ }^{2}$ Quantitative Software Developer-Stochastic Logic, Dhaka, Bangladesh \\ ${ }^{3}$ Associate Professor-Department of C.S.E., BUET, Dhaka, Bangladesh \\ E-mail: \{ashfaque, atiqul.islam\}@csebuet.org,mahmudanaznin@cse.buet.ac.bd \\ Received June 18, 2011; revised July 21, 2011; accepted July 31, 2011
}

\begin{abstract}
Target tracking is considered as one of the cardinal applications of a wireless sensor network. Tracking multiple targets is more challenging than tracking a single target in a wireless sensor network due to targets' movement in different directions, targets' speed variations and frequent connectivity failures of low powered sensor nodes. If all the low-powered sensor nodes are kept active in tracking multiple targets coming from different directions of the network, there is high probability of network failure due to wastage of power. It would be more realistic if the tracking area can be reduced so that less number of sensor nodes will be active and therefore, the network will consume less energy. Tracking area can be reduced by using the target's kinematics. There is almost no method to track multiple targets based on targets' kinematics. In our paper, we propose a distributed tracking method for tracking multiple targets considering targets' kinematics. We simulate our method by a sensor network simulator OMNeT++ and empirical results state that our proposed methodology outperforms traditional tracking algorithms.
\end{abstract}

Keywords: Wireless Sensor Network, Multiple Targets, Tracking, Target Kinematics

\section{Introduction}

Now-a-days, from military applications (battlefield surveillance and intelligence data acquisition), the use of sensor networks has extended to many civilian, industrial and environmental applications [1-2]. Among the diversified applications of sensor networks, target detection is one of the most important applications. In this context, the primary tasks of a sensor network are targets 'identification and classification, data acquisition, targets' tracking or localizing and data transmission over multi-hop networks [3]. Local information collected from sensor nodes in a particular time frame is used to obtain the global information regarding the location of the target. But due to power constraint and frequent connectivity failure of a sensor network, energy-efficient target tracking is a very challenging problem in the domain of sensor networks. Not only we need to ensure the service quality of the network, but also energy consumption should also be minimized. A popular method to optimize energy consumption in target tracking is the minimization of tracking area; the region which is reachable by a target from its current position with a certain speed. Previous works such as $[4,5]$ employ this approach to track targets efficiently. But they do not consider multiple types of targets at a single time. In practical scenario, a network has to detect multiple types of targets in a certain time frame. For example, in border surveillance, sensor nodes have to detect multiple targets such as wheeled vehicles or tracked vehicles such as tanks, or biological entities (human, animals). A target tracking methodology is desired to track all targets efficiently, regardless of their types or numbers. In our paper, we propose a novel, distributed, energy efficient multiple targets tracking method which includes target classification, data acquisition and transmission.

The core duties of detection, classification, tracking and identity management, are devolved into three types of sensor nodes, instead of confining into one. We use the concept of target kinematics [6] in tracking multiple targets to reduce the tracking area. It is not possible for a target (tracked or wheeled) to traverse every portion of its tracking area, because of its kinematics properties such as steering angle and speed. If we can reduce the 
tracking area, we can save energy consumption of the whole network as fewer number of sensor nodes of the tracking area are active. We have tried to reduce the tracking area using heuristics based on overlapping criteria. We have considered two ways of how these tracking areas can overlap: one-one and one-two overlapping. In the case of one-one overlapping, the tracking area of one target simultaneously overlaps with one and only one tracking area of one single target. In the case of one-two overlapping, the tracking area of one target simultaneously overlaps with the tracking area of two targets. We have provided heuristics and later explained those through illustrative figures. We have simulated our algorithm using a standard sensor network simulator OM$\mathrm{NeT}++[7]$ to validate our method. Our proposed method outperforms the traditional circular tracking area based approaches in $[4,5]$. The remainder of this paper is organized as follows: section 2 describes the related research work. In section 3 , we give the preliminaries. In section 4, we describe our tracking algorithm in details. In section 5, we report the experimental results and empirical analysis in form of tables and graphs. Finally, section 6 gives the summary of our work with a direction of future extension.

\section{Related Work}

In this section, we discuss some related research work. Bar-Shalom et al. propose a centralized tracking method where each sensor node transmits its sensing information towards a processing node which acts as a central processor and fuses the report collected from all sensing nodes [8]. Then, that processing node performs a long distance transmission to the base station. But, this approach suffers from network latency and synchronization problem, consumes huge amount of bandwidth and eventually introduces a single point of failure. Besides, the central node will be overloaded with computation.

In most of the sensor network applications, scalability is an essential requirement also [3]. It can be achieved by distributed tracking methods. In [4], Zhang et al. provide a distributed, tree based algorithm which they call $D y$ namic Convoy Tree based Collaboration (DCTC), where the sensor nodes track a single target. As a target moves along its trajectory, the tree formed by the sensor nodes, is continually reconfigured. Some nodes are added along the predicted path and are pruned if they are not required. Unfortunately, the construction of this convoy tree puts an additional computational overhead over the sensor nodes and it is almost impractical to implement.

In [9], Sikdar et al. use a linear prediction model to track a single target. This prediction model performs tracking based on the previous two observations. Even though the model can be extended to predict higher order predictions; these approaches require that all the sensor nodes to be kept alive, which eventually minimizes the lifetime of the network.

MCTA (Minimal Contour Tracking Algorithm), proposed by Tian He et al. in their paper [5], is also another centralized approach to track multiple targets. In their method, each sensor node is assigned to track a particular type of target which is a four-wheeled vehicle and sensors nodes send tracking information to the base station. Their computation is only applicable for tracking a certain type of targets, at a time. If there are other types of targets such as a tank or any biological entity passing through the network field, their algorithm will not be able to track. Therefore, for this approach to work properly, all possible target entities and their kinematics properties must be known.

\section{System Architecture and Preliminaries}

In this section, we describe our assumptions and definitions which are relevant to our work. From the perspective of energy levels, we assume that the network consists of two types of sensor nodes. A large portion consists of typical low powered sensor nodes and a small number of nodes with higher energy level are randomly deployed. The high energetic nodes provide better connectivity support [10]. According to responsibilities, we classify the sensor nodes in to three categories namely, Boundary Node (BN), Worker Node $(W N)$ and Computational Node $(C N)$. The sensor nodes that lie on the boundary of the network field are called $B N$. We call the high energetic nodes $C N$, as it will perform the necessary calculations for detection, classification, tracking and sleep-wake scheduling of the low powered sensor nodes. Each $C N$ is capable to relay target information to the base station or to other $C N \mathrm{~s}$ [10]. A $W N$ senses and transfers the target information. The whole tracking field is mapped into Voronoi polygons with the $\mathrm{CN}$ as the Voronoi center [11-13]. We call each Voronoi polygon a cell. Figure 1 describes a typical scenario of the network field. Targets can enter into the network field from any point on the boundary. There may be four types of targets: tracked vehicle, wheeled vehicle, biological entity such as human being and animal.

The $B N \mathrm{~s}$ are dedicated to target detection only. $W N \mathrm{~s}$ track targets and send information to the $C N$. At least three active $W N$ s are required to track a target according to triangulation property [14]. $W N \mathrm{~s}$ are responsible for providing tracking information and their energy is preserved with the help of target kinematics. We prefer to save $B N \mathrm{~s}$ ' energy to prevent single point of failure [15] in the sensor network. If all the $B N \mathrm{~s}$ become out of power 


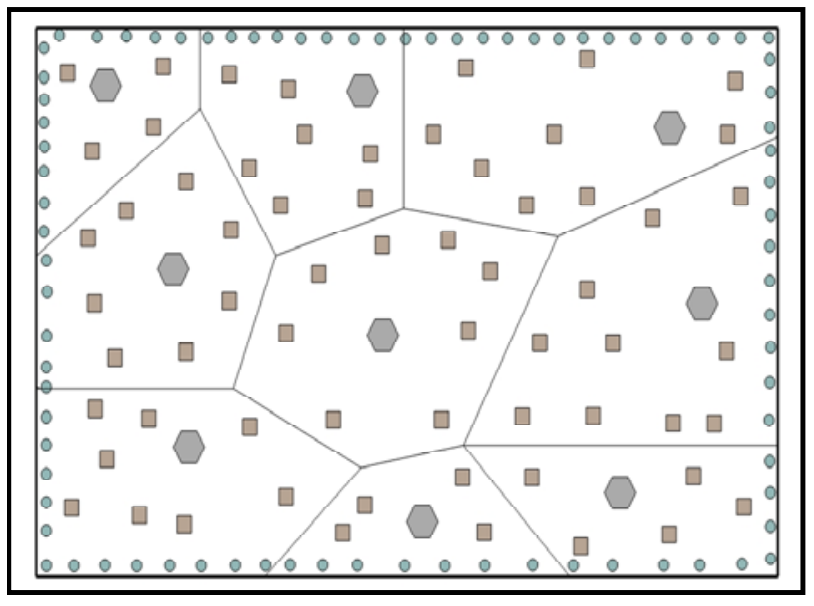

Figure 1. Proposed sensor network. Circles indicate the BNs along the boundary of the tracking field, squares indicate the WNs and the hexagons indicate the $C N s$.

near about the same time, the presence of targets will not be detected and detection failure rate will increase. This priority based task assignment to the sensor nodes shows an improvement in network energy consumption which is observed by empirical analysis later in section 6 . Focusing on energy preserving issue, we assume that we have only one layer of $B N \mathrm{~s}$. Now, we define some terms that are needed to explain our tracking method.

Definition A. Refresh Time. The longevity of the tracking area is defined as refresh time which implies that the old tracking area be replaced with the new tracking area according to the target's movement at every refresh time.

Definition B. Traverse Region. is the circular tracking area where the target can visit with its current position, speed and direction during refresh time.

Definition C. Polygon of Interest. By applying target kinematics a certain portion of the Traverse Region is obtained where the target is unable to reach [5]. Omitting the unnecessary tracking area from the Traverse Region results the Polygon of Interest for a particular refresh time.

Definition D. Overlapped Area of Interest. The common region covered by two or more overlapping Polygon of Interests is defined as Overlapped Area of Interest. This situation occurs when more than one target come close.

\section{Multiple Targets` Tracking using Polygon of Interest Algorithm (MTTP)}

After detecting targets, to classify targets we use a linear classifier classify the targets described in [16]. According to the classification decision provided by the linear classifier, our algorithm MTTP, uses tracking schemes on the basis of target kinematics [6]. Intuitive mathematical modeling provides the kinematic properties of the targets present in cells. The details of the detection, classification and mathematical models are available in [16]. The Polygon of Interests is computed by each $C N$ in the relevant cell where the target is tracked.

\section{Algorithm 1 MTTP (tracking_field_info)}

1. for each $C N, \zeta_{i}$ in $\chi$ do

2. $\Delta_{i}=$ Create_Self_Cell $\left(\zeta_{i}\right)$;

\{Creating cell and eventually partition the whole tracking field into cells dynamically.\}

3. Perform_Detection_Job (detection signal from $B N \mathrm{~s}$ );

$\{$ Differentiate between a false alarm and a real target appearance.\}

4. Classification_Job (acoustic features from $B N \mathrm{~s}$ );

$\{$ The classifier will classify the targets present in cell $\Delta_{i}$.

5. $\quad \lambda_{i}=$ Know_WNs_Cell $\left(\Delta_{i}\right)$

6. Get_Kinematics_Info (tracking_info

from each active $\lambda_{i}$ );

(Speed, velocity, time for sampling, angle respect to the co-ordinate system will be obtained from this procedure.\}

7. $\quad t=$ Get_Refresh_Time (v);

8. $\gamma_{t}^{p}=$ Form_Polygon_of_Interest $(X, Y, t$, $\theta, v)$;

\{Using the position, orientation and speed of a target, this module creates the corresponding Polygon of Interest. \}

9. Assign_WN_Identity $\left(\lambda_{i}, \gamma_{t}^{p}\right)$;

10. $\quad \kappa=$ Find_Total $\left(\Delta_{i}, \gamma_{t}^{p}\right)$;

$\{$ Determines the total number of Polygon of Interests in the cell.\}

11. Overlap $(t, \kappa)$;

12. Go to 6

13. end for

Algorithm 2 ASSIGN_WN_IDENTITY $\left(\lambda_{i}^{j}, \gamma_{t}^{p}\right)$

1. for each $\lambda_{i}{ }^{j} \varepsilon \lambda_{i}$ do

2. if $\left(\right.$ Check_Inclusion $\left.\left(\lambda_{i}^{j}, \gamma_{t}^{p}\right)\right)$ then

3. $\quad$ Activate $\left(\lambda_{i}^{j}\right)$;

4. Assign_Identity $\left(\lambda_{i}^{j}, \gamma_{t}^{p}\right)$;

5. else

6. $\quad$ Skip

7. end if

8. end for

Let, $\zeta_{i}$ be the $i^{\text {th }} C N$, acting as the head of $c e l l \Delta_{i}$, in a 
tracking field $\chi$, where, $\zeta_{i} \varepsilon \zeta$ is the set of $C N \mathrm{~s}$ in the tracking field $\chi$, and $\Delta_{i} \varepsilon \Delta$, is the set of cells formed in the tracking field, $\chi$. Here we consider the total network which will track multiple targets as tracking field. We also assume $\lambda_{i}^{j}$ as the $j^{\text {th }} \mathrm{WN}$ for $i^{\text {th }}$ cell $\Delta_{i}$, in the tracking field $\chi$, where $\lambda_{i}^{j} \varepsilon \lambda_{i}$, the set of the $W N \mathrm{~s}$ in $i^{\text {th }}$ cell $\Delta_{i}$ and true for all $i=1 . n$ in $\chi$. Here, $n$ is also the total number of active cells in the tracking field $\chi$, as the number of active cells will be as same as the number of $C N \mathrm{~s}$ in the tracking field $\chi$. Let us assume $k$ to be the total number of Polygon of Interests present in the $i^{\text {th }}$ cell, $\Delta_{i}$, at time $t$. Let, $\gamma_{t}^{p}$ be the corresponding Polygon of Interest of target $p$ at any time $t$, in tracking field $\chi$ and $\mu_{t}^{p}$, be the corresponding area covered by the Polygon of Interest $\gamma_{t}^{p}$ at time $t$. The number of active $W N \mathrm{~s}$ in a Polygon of Interest $\gamma_{t}^{p}$ is $\eta_{t}^{p}$, for target $p$. We also call $\mu_{t}^{p, k}$ to be the Overlapped Area of Interest between $\mu_{t}^{p}$ and $\mu_{t}^{k}$, the corresponding area of Polygon of Interests, $\gamma_{t}^{p}$ and $\gamma_{t}^{k}$ respectively.

Algorithm 1 is the main module of MTTP. It provides all the provisions to detect, classify and track multiple targets. Algorithm 2 works as a sub-module to give the identity of the $W N \mathrm{~s}$ in a Polygon of Interest. Algorithm 3 is associated with overlap detection and calls the corresponding modules. Algorithm 4, 5 and 6 are completely responsible for overlap operations.

\section{Algorithm 3 Overlap $(t, \kappa)$}

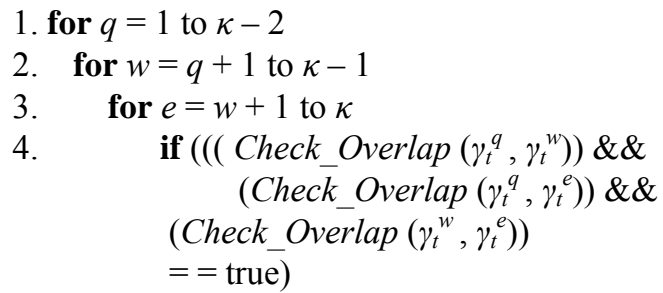

5. Get overlapped region of the three Poly gon of Interests, $\gamma_{t}^{q}, \gamma_{t}^{w}, \gamma_{t}^{e}$; let it be denoted as $\gamma_{t}^{q, w, e}=\gamma_{t}^{r}$.

6. Get overlapped region of the two Polygon of Interests, $\gamma_{t}^{q}, \gamma_{t}^{w}$; let it be denoted as $\gamma_{t}^{q, w}$

7. Get overlapped region of the two Polygon of Interests, $\gamma_{t}^{q}, \gamma_{t}^{e}$; let it be denoted as $\gamma_{t}^{q, e}$

8. Get overlapped region of the two Polygon of Interests, $\gamma_{t}^{w}, \gamma_{t}^{e}$; let it be denoted as $\gamma_{t}^{\text {w,e }}$

9. $\quad$ Simultaneous Overlapping of Three Polygon of Interests $\left(\gamma_{t}^{q}, \gamma_{t}^{w}, \gamma_{t}^{e}, \gamma_{t}^{r}\right.$, $\left.\gamma_{t}^{q, w}, \gamma_{t}^{q, e}, \gamma_{t}^{w, e}\right)$

10. else

11. Take no action. Report that simultaneous overlapping of

\author{
three Polygon of Interests have \\ not occurred. \\ 12. end if \\ 13. end for \\ 14. end for \\ 15. end for \\ 16. for $q=1$ to $\kappa-1$ \\ 17. for $w=q+1$ to $\kappa$ \\ 18. if (Check_Overlap $\left.\left(\gamma_{t}^{q}, \gamma_{t}^{w}\right)\right)$ \\ 19. Simultaneous Overlapping of Two \\ 20. else \\ Polygon of Interests $\left(\gamma_{t}^{q}, \gamma_{t}^{w}\right)$
}

Take no action. Report that simultaneous overlapping of two Polygon of Interests have not occurred.

\section{1. end if \\ 22. end for \\ 23. end for}

For making $W N \mathrm{~s}$ awake or asleep, we use the concept of Polygon of Interest with the help of point in polygon algorithm [13]. In case of overlapping, Overlapped Polygon of Interests, are reconstructed on the basis of mutual relativity, in size and properties of the Polygon of Interests. We explore the internal details of our algorithm in this section.

Algorithm 4 Simultaneous Overlapping of Three Polygon of Interests $\left(\gamma_{t}^{a}, \gamma_{t}^{b}, \gamma_{t}^{c}, \gamma_{t}^{d}, \gamma_{t}^{e}, \gamma_{t}^{f}, \gamma_{t}^{g}\right)$

1. Get the area of the following contours $\gamma_{t}^{a}, \gamma_{t}^{b}, \gamma_{t}^{c}$ and $\gamma_{t}^{d}$. Let it be denoted as $\mu_{t}^{a}, \mu_{t}^{b}, \mu_{t}^{c}$ and $\mu_{t}^{d}$ respectively.

2. Compute $\gamma_{t}^{a 1}$, which will be the portion of $\gamma_{t}^{a}$ included in $\gamma_{t}^{d}$.

3. Compute $\gamma_{t}^{b 1}$, which will be the portion of $\gamma_{t}^{b}$ included in $\gamma_{t}^{d}$.

4. Compute $\gamma_{t}^{c 1}$, which will be the portion of $\gamma_{t}^{c}$ included in $\gamma_{t}^{d}$.

5. Compute $\gamma_{t}^{e 1}$, which will be the portion of $\gamma_{t}^{e}$ included in $\gamma_{t}^{d}$.

6. Compute $\gamma_{t}^{f 1}$, which will be the portion of $\gamma_{t}^{f}$ included in $\gamma_{t}^{d}$.

7. Compute $\gamma_{t}^{g 1}$, which will be the portion of $\gamma_{t}^{g}$ included in $\gamma_{t}^{d}$.

8. Compute $\mu_{t}^{a 1}, \mu_{t}^{b 1}$ and $\mu_{t}^{c 1}$ from $\gamma_{t}^{a 1}, \gamma_{t}^{b 1}$ and $\gamma_{t}^{c 1}$ respectively.

9. Get the updated area $\mu_{t}^{a}, \mu_{t}^{{ }^{b}}$ and $\mu_{t}^{{ }^{c}}$ using $\mu_{t}^{{ }^{a}}=$ $\mu_{t}^{a}-\mu_{t}^{a 1}, \mu_{t}^{{ }^{b}}=\mu_{t}^{b}-\mu_{t}^{b 1}$ and $\mu_{t}^{c}=\mu_{t}^{c}-\mu_{t}^{c 1}$ respectively and update $\gamma_{t}^{a}, \gamma_{t}^{b}, \gamma_{t}^{c}$ as $\gamma_{t}^{\wedge a}, \gamma_{t}^{{ }^{b}}$ and $\gamma_{t}^{c c}$ respectively.

10. Get the updated area $\mu_{t}^{{ }^{e}}, \mu_{t}^{{ }^{f}}$ and $\mu_{t}^{{ }^{g}}$ using $\mu_{t}^{\cdot e}=$ 
$\mu_{t}^{e}-\mu_{t}^{e 1}, \mu_{t}^{f}=\mu_{t}^{f}-\mu_{t}^{f 1}$ and $\mu_{t}^{\mathrm{g}}=\mu_{t}^{g}-\mu_{t}^{g 1}$ respectively and update $\gamma_{t}^{e}, \gamma_{t}^{f}, \gamma_{t}^{g}$ as $\gamma_{t}^{\cdot e}, \gamma_{t}^{\prime f}$ and $\gamma_{t}^{\cdot g}$ respectively.

11. Calculate $\eta_{t}{ }^{1}, \eta_{t}{ }^{2}$ and $\eta_{t}^{3}$ from $\gamma_{t}^{{ }_{t}^{a}}, \gamma_{t}^{{ }_{t}^{b}}$ and $\gamma_{t}^{{ }^{c}}$ respectively.

12. If $\left(\eta_{t}{ }^{1}=>3\right)$

13. Individual Minimization $\left(\gamma_{t}^{\backslash a}, \gamma_{t}^{` e}, \gamma_{t}^{\vee f}\right)$

14. Else

15. Preserve $\gamma_{t}^{a}$. No minimization will take place. Return $\gamma_{t}^{a}$

16. If $\left(\eta_{t}^{2}=>3\right)$

17. Individual Minimization $\left(\gamma_{t}^{\cdot b}, \gamma_{t}^{\cdot e}, \gamma_{t}^{\cdot g}\right)$

18. Else

19. Preserve $\gamma_{t}^{b}$. No minimization will take place. Return $\gamma_{t}^{b}$

20. If $\left(\eta_{t}{ }^{3}=>3\right)$

21. Individual Minimization $\left(\gamma_{t}^{\cdot c}, \gamma_{t}^{\vee f}, \gamma_{t}^{\prime g}\right)$

22. Else

23. Preserve $\gamma_{t}^{c}$. No minimization will take place. Return $\gamma_{t}^{c}$

For simplicity, to describe the overlapping issue we consider two Polygon of Interests of two targets at a certain time $t$. We check overlapping, by relatively detecting the inclusion of one Polygon of Interest into another. The $W N$ s remain completely static during tracking. The Polygon of Interests is reconstructed in such a way that each of the Polygon of Interest possesses the sufficient number of $W N$ s to track the targets. Let two Polygon of Interests be, $\gamma_{t}^{a}$ and $\gamma_{t}^{b}$ where $\mu_{t}^{a}$ and $\mu_{t}^{b}$, are respectively their areas. The number of $W N \mathrm{~s}$ in $\gamma_{t}^{a}$ and $\gamma_{t}^{b}$ is respectively denoted as, $\eta_{t}{ }^{a}$ and $\eta_{t}^{b}$. We check the inclusion of $\gamma_{t}^{b}$, which we call the Compared Polygon of Interest into the Comparing Polygon of Interest, $\gamma_{t}^{a}$. If the inclusion is true we consider the two Polygon of Interests to be overlapped. Then we calculate the area common between the Polygon of Interests, $\gamma_{t}^{a}, \gamma_{t}^{b}$ and denote it as $\mu_{t}^{a, b}$. We then adjust $\mu_{t}^{b}$, which represents the area of Compared Polygon of Interest $\gamma_{t}^{b}$, by subtracting $\mu_{t}^{a, b}$ from it. $\eta_{t}^{a}, \eta_{t}^{b}, \eta_{t}^{a, b}$ is also adjusted accordingly. We illustrate three cases based on the value of $\eta_{t}^{b}$.

Algorithm 5 Individual Minimization $\left(\gamma_{t}{ }^{1}, \gamma_{t}{ }^{2}, \gamma_{t}{ }^{3}\right)$

1. Compute $\gamma_{t}^{x}$ as the area of $\gamma_{t}^{1}$ which is included in the area of $\gamma_{t}^{2}$

2. Compute $\gamma_{t}^{y}$ as the area of $\gamma_{t}{ }^{1}$ which is included in the area of $\gamma_{t}^{3}$

3. Compute $\mu_{t}^{x}$ and $\mu_{t}^{y}$ from $\gamma_{t}^{x}$ and $\gamma_{t}^{y}$ respectively

4. Calculate $\gamma_{t}^{1}$ as $\gamma_{t}^{0}$ using $\mu_{t}^{0=} \mu_{t}^{1}-\mu_{t}^{x}$

5. Calculate $\gamma_{t}^{1}$ as $\gamma_{t}^{9}$ using $\mu_{t}{ }^{9}=\mu_{t}{ }^{1}-\mu_{t}^{y}$

6. Calculate $\eta_{t}{ }^{9}$ and $\eta_{t}{ }^{0}$.

7. If $\left(\eta_{t}{ }^{9}=>3 \| \eta_{t}{ }^{0}=>3\right)$

8. $\quad$ Calculate $\eta_{t}^{\text {Factor }}=\operatorname{Minimum}\left(\eta_{t}{ }^{9}, \eta_{t}{ }^{0}\right)$
9. $\quad$ If $\eta_{t}^{\text {Factor }}=>3$

10. Compute $\gamma_{t}^{\text {Factor }}$ in correspondence to $\eta_{t}^{\text {Factor }}$

11. Preserve $\gamma_{t}^{\text {Factor }}$

12. Else

13. Calculate $\eta_{t}^{\text {Factor }}=\operatorname{Maximum}\left(\eta_{t}{ }^{9}, \eta_{t}{ }^{0}\right)$

14. Compute $\gamma_{t}^{\text {Factor }}$ in correspondence to $\eta_{t}^{\text {Factor }}$

15. Preserve $\gamma_{t}^{\text {Factor }}$

16. Return $\gamma_{t}^{\text {Factor }}$

17. Else

18. Preserve $\gamma_{t}^{1}$

19. Return $\gamma_{t}^{1}$

In the following illustration we study how Overlapped Area of Interests effect the tracking decision policy while tracking multiple targets. Larger Overlapped Area of Interest between two Polygon of Interests results in smaller number of $W N \mathrm{~s}$ in that Polygon of Interest.

$C A S E$ 1. Here, we describe a certain situation that can take place in the sensor network when two targets are very close to each other. In this case, the corresponding Polygon of Interests overlap each other by a large amount. Figure 2 is the illustration of CASE 1. Here in Figure 2(a), $X$ and $Y$ represent the Polygon of Interests of two different targets, where $X$ and $Y$ are the Comparing Polygon of Interest and Compared Polygon of Interest respectively. According to our algorithm, size of $Y$ is reduced by the overlapped portion after detecting overlapping. Therefore, the number of $W N \mathrm{~s}$ included in Compared Polygon of Interest, but not in Overlapped Area of Interest, is zero as indicated in Figure 2(b) (no WNs in $Y$ ).

Algorithm 6 states that when the number of $W N \mathrm{~s}$ in the Compared Polygon of Interest, i.e., $\eta_{t}^{b}$ is less than or equal to one, the number of $W N \mathrm{~s}$ in the Comparing Polygon of Interest is divided by two and the similar number of $W N \mathrm{~s}$ is allocated to the both, Comparing Polygon of Interest ( $X$ in Figure 2(c)) and Compared Polygon of Interest ( $X$ in Figure 2(c)).

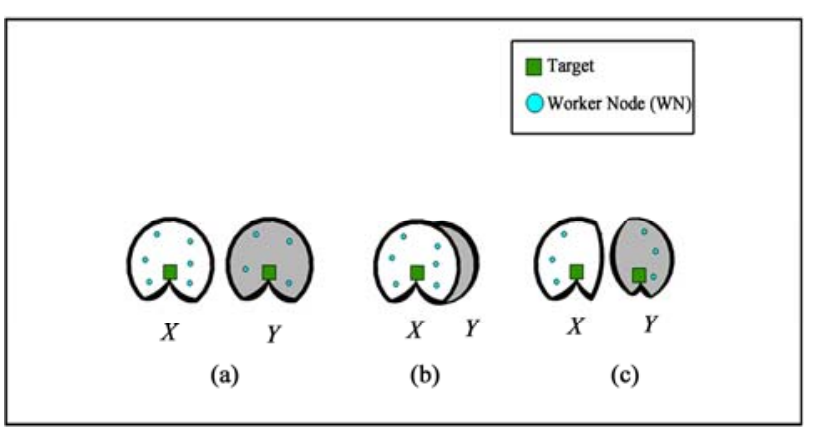

Figure 2. Illustration of Case 1. 
Algorithm 6 Simultaneous Overlapping of Two Polygon of Interests $\left(\gamma_{t}^{q}, \gamma_{t}^{l}\right)$

1. Get the overlapped area $\mu_{t}^{q, l}$ from $\mu_{t}^{q}$ and $\mu_{t}^{l}$.

2. Update $\mu_{t}^{l}$ using $\mu_{t}^{l}=\mu_{t}^{l}-\mu_{t}^{q, l}$ and adjust $\eta_{t}^{l,}$ accordingly.

3. if $\eta_{t}^{l}<=1$ then

4. $\quad \eta_{t}^{q}=$ Floor $\left(\eta_{t}^{q} / 2\right)$

5. end if

6. if $\eta_{t}^{l}==2$ then

7. $\quad \eta_{t}^{q}=$ Floor $\left(\eta_{t}^{q} / 3\right)$

8. end if

9. Update $\gamma_{t}^{q}$ such that $\eta_{t}^{q}=\eta_{t}^{q}-\eta_{t}^{q}$

10. Update $\gamma_{t}^{l}$ such that $\eta_{t}^{l}=\eta_{t}^{l}+\eta_{t}^{\prime l}$

CASE 2. Here, the margin of overlap among two targets is not as large in CASE 1. In Figure 3(a), $X$ and $Y$, represent the Polygon of Interest of two targets. As they come close to each other, their respective Polygon of Interests overlap. Necessary reduction is done from the two Polygon of Interests and the number of $W N \mathrm{~s}$ is calculated. According to Figure 3(b), the number of $W N \mathrm{~s}$ in Compared Polygon of Interest $(Y)$ but not in Overlapped Area of Interest, is two. Algorithm 6 states when $\eta_{t}^{b}$, the number of $W N \mathrm{~s}$ in the present Compared Polygon of Interest is equal to two, the number of active $W N \mathrm{~s}$ in the Comparing Polygon of Interest $\gamma_{t}^{a}$, is divided by three and the floor of the quotient is taken. We then add this number of $W N \mathrm{~s}$ to $\eta_{t}^{b}$. Subtraction from $\eta_{t}^{a}$ is done by the same amount. The reallocation of $W N$ s sets up the two Polygon of Interests track their corresponding targets.

CASE 3. CASE 3 is also very simple. As the overlap between the two Polygon of Interests are low, no significant change occurs in the two Polygon of Interests as stated in Figure 4. When the number of active $W N \mathrm{~s}$ in the Polygon of Interest $\eta_{t}^{b}$ is equal to or greater than three we consider it sufficient to track target with proper id management. The value of $\eta_{t}^{a}$ and $\eta_{t}^{b}$ is kept unchanged.

CASE 4. We use Figures 5, 6 and 7 to explore the mechanisms of our proposed methodology to describe a scenario where three Polygon of Interests are overlapping. There are three Polygon of Interests $X, Y$ and $Z$ according to Figure 5(a). As Figure 5(b) states, there are three Polygon of Interests namely; $X, Y$ and $Z$ are overlapped with each other. As the condition (according to Algorithm 3, line 4) to check overlap is fulfilled, according to our algorithm (according to Algorithm 3, line 5) first of all we need to get the common area of these three Polygon of Interests.

Let this Polygon of Interest be named as $D$. We will also calculate the Overlapped Area of Interest, $A$ between $X$ and $Y$; Overlapped Area of Interest, $B$ between $X$ and $Z$; Overlapped Area of Interest, $C$ between $Y$ and $Z$.

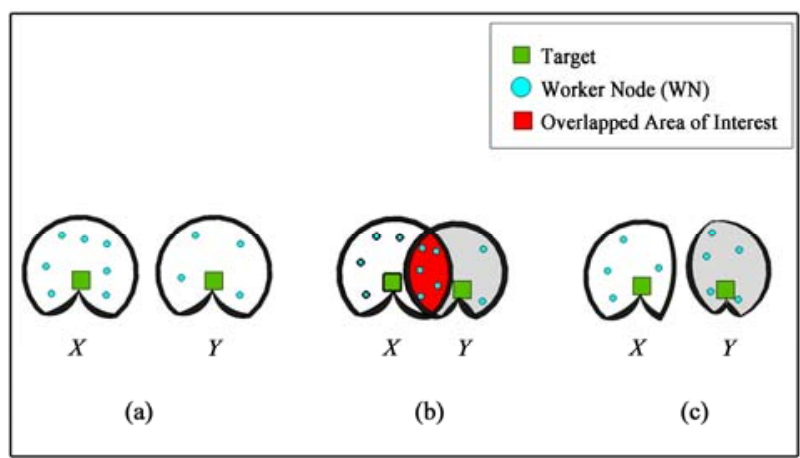

Figure 3. Illustration of Case 2.

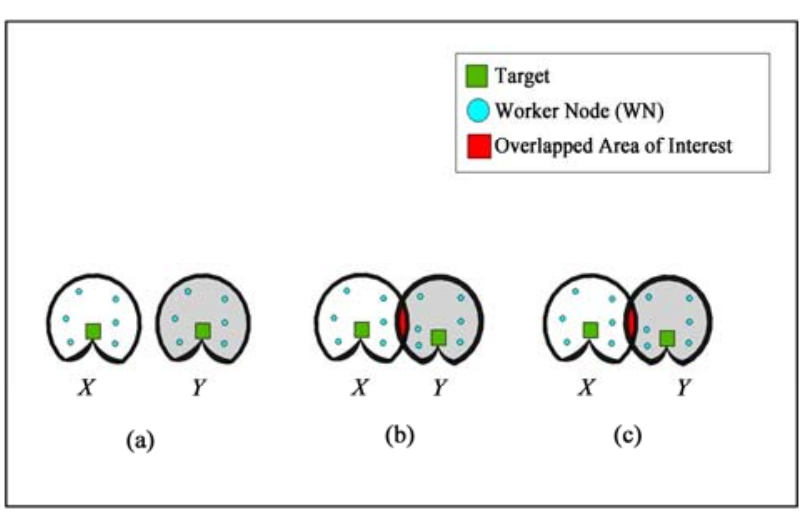

Figure 4. Illustration of Case 3.

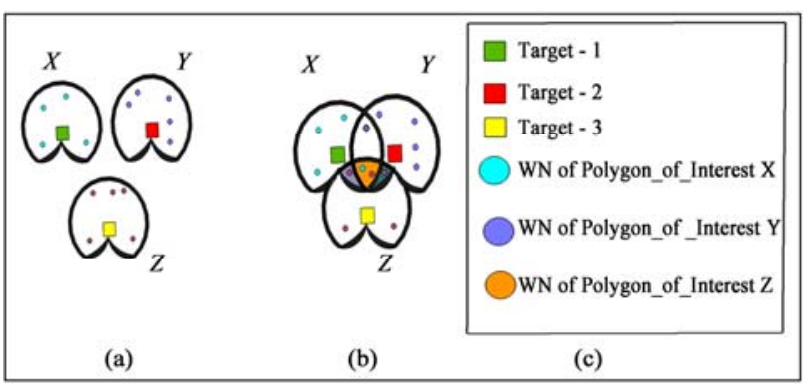

Figure 5. Illustration of CASE 4.

Now for each Polygon of Interest $X, Y$ and $Z$ the algorithm Simultaneous Overlapping of Three Polygon of Interests is called. First of all the area of $X, Y, Z$ and $D$ will be calculated; let them be named respectively as $\operatorname{Area}(X)$, Area $(Y)$, Area $(Z)$ and $\operatorname{Area}(D)$ (according to Algorithm 4, line 1). Now we have to find that portion of the Polygon of Interest $X$ which is included in $D$. Let it be named as $X 1$. The same procedure is also applied to $Y$ and $Z$ and the corresponding area is named as $Y 1$ and $Z 1$ respectively (according to Algorithm 4, line 2, 3, 4). We know compute $X^{\prime}, Y^{\prime}$ and $Z^{\prime}$ as $X^{\prime}=X-X 1$ (shown in Figure 6(a)), $Y^{\prime}=Y-Y 1$ (shown in Figure 6(b)) and $Z^{\prime}=$ $Z-Z 1$ (shown in Figure 6(c)). $X^{\prime}, Y^{\prime}$ and $Z^{\prime}$ is shown in Figure 6(d) respectively. 
Now we will calculate the Overlapped Area of Interest between the Polygon of Interests $X$ and $Y, X$ and $Z$ and $Y$ and $Z$ and name them respectively as $A, B$ and $C$. Now we have to find that portion of $A$ which is included in $D$; we name it as $D 1$. In the same manner we calculate $D 2$ for $B$ and $D 3$ for $C$. We will then calculate $A{ }^{\wedge}, B^{`}$ and $C^{\wedge}$ using $A^{`}=A-D 1, B=B-D 2$ and $C^{`}=C-D 3$ (according to Algorithm 4, line 9). Result of these calculations are shown in Figure 6(e) and it is evident from the figure that then number of $W N \mathrm{~s}$ in the Polygon of Interests $A$; $B$ ' and $C^{\prime}$ is one for each of the Polygon of Interests.

We will now calculate the number of $W N \mathrm{~s}$ in $X^{\prime}, Y^{\prime}$ and $Z^{`}$ and we denote them as $n\left(X^{\prime}\right), n\left(Y^{\prime}\right)$ and $n\left(Z^{\prime}\right)$ respectively (according to Algorithm 4, line 12). According to Figure 5(f), 5(g) and 5(h), $n\left(X^{\prime}\right)=3, n\left(Y^{\prime}\right)=$ 5 and $n(Z)=4$. As the numbers of $W N$ s in each of the minimized Polygon of Interests are greater than or equal to 3 , according to our algorithm the condition to call algorithm Individual Minimization is fulfilled.

The algorithm Individual Minimization will be called (according to Algorithm 4, line 13) with the following parameters: Polygon of Interest $X^{\prime}, A^{`}$ and $B$ '.

The algorithm Individual Minimization will be called (according to Algorithm 4, line 17) with the following parameters: Polygon of Interest $Y^{\prime}, A^{\prime}$ and $C^{\prime}$.

The algorithm Individual Minimization will be called (according to Algorithm 4, line 21) with the following parameters: Polygon of Interest $Z Z^{\prime}, B^{`}$ and $C^{\prime}$.

Calling algorithm Individual Minimization (Algorithm 5 ) with the following parameters, Polygon of Interest $X$, $A$ 'and $B$ :

First of all, we will calculate the portion of $X^{\prime}$ which is included in $A^{\text {' }}$ (according to Algorithm 5, line 1); we name this area as $A 1^{\text {. }}$. Then we calculate the portion of $X^{\prime}$ which is included in $B^{`}$ (according to Algorithm 5, line 2) and name this area as $B 1$. We will create two Polygon of Interests $X 0$ and $X 9$ using $X 0=X^{`}-A 1^{`}$ (according to Algorithm 5, line 4) and $X 9=X^{`}-B 1^{`}$ (according to Algorithm 5, line
5). We illustrate the calculation of $X 0$ in Figure 7(a1) and the calculation of $X 9$ in Figure 7(a2). We calculate the number of $W N \mathrm{~s}$ of $X 0$ and $X 9$ and denote them respectively as $n(X 0)$ and $n(X 9)$. It is evident that $n(X 0)=3$ and $n(X 9)=3$. We now need to find the Polygon of Interest $X F a c t o r$, whose number of $W N$ s included in the Polygon of Interest is denoted as $n(X F a c t o r)$ and which will be the final result of the algorithm Individual Minimization. According to the figure Minimum ( $n(X 0)$, $n(X 9))=3$. So, $n($ XFactor $)=3$ (according to Algorithm 5, line 8). As $n$ (XFactor) fulfills the condition so Polygon of Interest XFactor as shown in Figure 7(a3) will be computed, preserved and will be returned (according to Algorithm 5, line 10).

Calling algorithm Individual Minimization (Algorithm $5)$ with the following parameters, Polygon of Interest $Y$, $A^{\prime}$ and $C^{\prime}$ :

First of all, we will calculate the portion of $Y^{\wedge}$ which is included in $A^{\prime}$; we name this area as $A 2{ }^{\prime}$. Then we calculate the portion of $Y^{\prime}$ which is included in $C^{\prime}$ and name this area as $C 2^{\circ}$. We update $Y 1$ as $Y 1=Y^{`}-A 2^{`}$ and $Y 2$ as $Y 2=Y^{\prime}-C 2^{\prime}$ which is shown in Figure 7(b1) and 7(b2). We calculate the number of $W N \mathrm{~s}$ of $Y 1$ and $Y 2$ and denote them respectively as $n(Y 1)$ and $n(Y 2)$. We now need to find the Polygon of Interest $Y F a c t o r$, whose number of $W N \mathrm{~s}$ included in the Polygon of Interest is denoted as $n$ (YFactor) and which will be the final result of the algorithm Individual Minimization. According to the figure Minimum ( $n(Y 1), n$ $(Y 2))=4$. So, $n($ YFactor $)=4$. As $n($ YFactor $)$ fulfills the condition so Polygon of Interest, YFactor will be computed, preserved and will be returned.

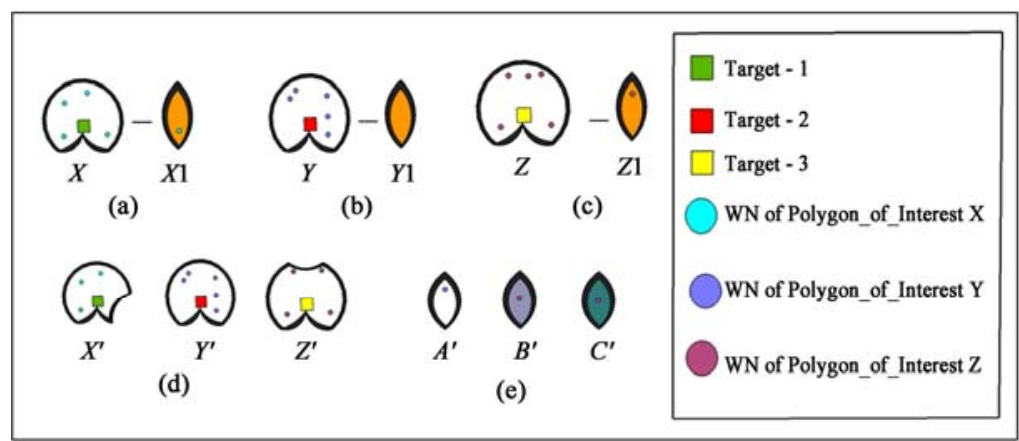

Figure 6. Illustration of CASE 4(Contd). 


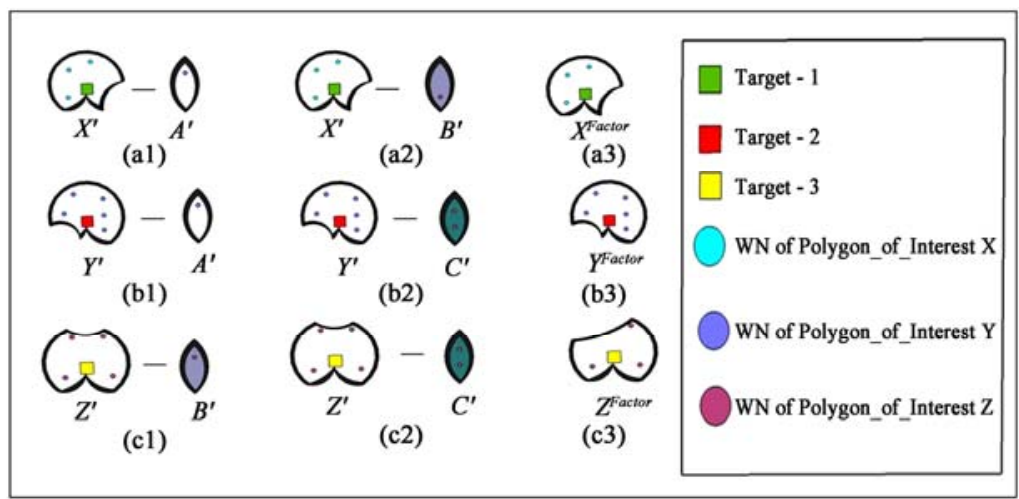

Figure 7. Illustration of CASE 4 (Contd.).

Calling algorithm Individual Minimization (Algorithm 5) with the following parameters, Polygon of Interest $Z$;, $B$ ' and $C$ :

First of all, we will calculate the portion of $Z$ which is included in $B$; we name this area as $B 3$ `. Then we calculate the portion of $Z$ ' which is included in $C$ 'and name this area as $C 3^{\prime}$. We update $Z 1$ as $Z 1=Z{ }^{`}-B 3^{`}$ and $Z 2$ as $Z 2=Z{ }^{`}-C 3^{`}$ which is shown in Figure 7(c1) and 7(c2). We calculate the number of $W N$ s of $Z 1$ and $Z 2$ and denote them respectively as $n(Z 1)$ and $n(Z 2)$. We now need to find the Polygon of Interest ZFactor, whose number of $W N$ s included in the Polygon of Interest is denoted as $n$ (ZFactor) and which will be the final result of the algorithm Individual Minimization. As $n(Z 1)=3$ and $n(Z 2)=3$, according to the figure Minimum $(n(Z 1), n(Z 2))=3$. So, $n($ XFactor $)=3$ (according to

Algorithm 5, line 8). As $n$ (XFactor) fulfills the condition (according to Algorithm 5, line 9) so Polygon of Interest XFactor as shown in Figure 7(a3) will be computed (according to Algorithm 5, line 10), preserved (according to Algorithm 5, line 11) and will be returned (according to Algorithm 5, line 16).

The algorithm Simultaneous Overlapping of Three Polygon of Interests can be reduced to describe the mechanisms when one Polygon of Interest overlaps with another Polygon of Interest, at a certain time. We call it the Simultaneous Overlapping of Three Polygon of Interests Reduced to Two algorithm denoted by Algorithm 7. We assume it will need at least three $W N$ s in one single Polygon of Interest to track one single target.

Algorithm 7: Simultaneous Overlapping of Three Polygon of Interests Reduced to Two $\left(\gamma_{t}^{a}, \gamma_{t}^{b}\right)$
1. Get the area of the Polygon of Interests $\gamma_{t}^{a}$ and $\gamma_{t}^{b}$. Let it be denoted as $\mu_{t}^{a}$ and $\mu_{t}^{b}$ respectively.

2. Compute the overlapped area between $\mu_{t}^{a}$ and $\mu_{t}^{b}$ and let it be denoted as $\mu_{t}^{c}$.

3. Compute $\gamma_{t}^{a 1}$, which will be the portion of $\gamma_{t}^{a}$ included in $\gamma_{t}^{c}$.

4. Compute $\gamma_{t}^{b 1}$, which will be the portion of $\gamma_{t}^{b}$ included in $\gamma_{t}^{c}$.

5. Compute $\mu_{t}^{a 1}$ and $\mu_{t}^{b 1}$ from $\gamma_{t}^{a 1}$ and $\gamma_{t}^{b 1}$ respectively.

6. Get the updated area $\mu_{t}^{{ }^{a}}$ and $\mu_{t}^{{ }_{t}^{b}}$ using $\mu_{t}^{{ }^{a}}=\mu_{t}^{a}-$ $\mu_{t}^{a 1}$ and $\mu_{t}^{{ }^{b}}=\mu_{t}^{b}-\mu_{t}^{b 1}$ respectively and update $\gamma_{t}^{a}$ and $\gamma_{t}^{b}$ as $\gamma_{t}^{{ }^{a}}$ and $\gamma_{t}^{{ }^{b}}$ respectively.

7. Calculate $\eta^{1}$ and $\eta^{2}$ from $\gamma_{t}^{{ }^{a}}$ and $\gamma_{t}^{{ }^{b}}$ respectively.

8. If $\left(\eta^{1}=>3\right)$

9. Preserve minimization. Return $\gamma_{t}^{{ }^{a}}$.

10. Else

11. Preserve $\gamma_{t}^{a}$. No minimization will take place. Return $\gamma_{t}^{a}$

12. If $\left(\eta^{2}=>3\right)$

13. Preserve minimization. Return $\gamma_{t}^{\circ b}$.

14. Else

15. Preserve $\gamma_{t}^{b}$. No minimization will take place. Return $\gamma_{t}^{b}$.

We describe some case studies below to further illustrate the algorithm.

$C A S E$ 5. Here, we describe a certain situation that can take place in the sensor network when two targets are close to each other. In this case, the corresponding Polygon of Interests overlap each other by a significant amount. Figure 8 is the illustration of CASE 5. Here in Figure 8(a), $X$ and $Y$ represent the Polygon of Interests of two different targets. In Figure 8(b) the two Polygon of Interests overlap. According to Algorithm 7, the Overlapped Area of Interest of the two Polygon of Interests, $X$ and $Y$ will be calculated. Let it be named as $Z$. Then the area of $X$ and $Y$ included in $Z$ will be calculated (Algorithm 7, line 5); let them be denoted as $X 1$ and $Y 1$ 
respectively. We will now calculate $X^{`}$ and $Y^{`}$ using $X^{`}=X$ $-X 1$ and $Y^{`}=Y-Y 1$ respectively (algorithm 7, line 6). The calculations to find $X^{`}$ and $Y^{`}$ is represented in Figure 8(c) and 8(d) respectively. Now we calculate the number of $W N \mathrm{~s}$ in $X^{\prime}$ and $Y^{`}$ and denote them as $n\left(X^{\prime}\right)$ and $n\left(Y^{\prime}\right)$. According to Figure 8(e), $n\left(X^{\prime}\right)=3$ and Figure 8(f), $n\left(Y^{\prime}\right)=$ 5.

Thus minimization will be preserved according to our algorithm (line 9 and line 13). Polygon of Interests $X^{\prime}$ and $Y$ is the final outcome of this study.

CASE 6. In this case, the corresponding Polygon of Interests overlaps each other by a small amount. Figure 9 is the illustration of CASE 6. Here in Figure 9(a), $X$ and $Y$ represent the Polygon of Interests of two different targets. Figure 9(b) represents the overlap between the two Polygon of Interests. According to our algorithm, first the Overlapped Area of Interest of the two Polygon of Interests namely, $X$ and $Y$ will be calculated. Let it be named as $Z$. Then the area of $X$ and $Y$ included in $Z$ will be calculated; let them be denoted as $X 1$ and $Y 1$ respectively. We will now calculate $X^{`}$ and $Y^{`}$ using $X^{`}=X-X 1$ and $Y^{`}=Y-Y 1$ respectively. Now we calculate the number of $W N \mathrm{~s}$ in $X^{\circ}$ and $Y^{`}$ and denote them as $n\left(X^{\prime}\right)$ and $n\left(Y^{`}\right)$. According to
Figure 9(c), $n\left(X^{\prime}\right)=3$ (Algorithm 7, line 7) and $n\left(Y^{\prime}\right)=$ 2.The minimization for Polygon of Interest $X$ will be preserved but minimization for Polygon of Interest $Y$ will not be preserved according to our algorithm 7 (line 15). We will restore Polygon of Interest $Y$ by adding $Y=Y^{`}+$ $Y 1$. The outcome of this case study are the Polygon of Interests are $X^{`}$ and $Y^{`}$ as indicated in Figure 9(e) and 9(f) respectively.

$C A S E$ 7. Figure 10 is the illustration of CASE 7. Here in Figure 10(a), $X$ and $Y$ represent the Polygon of Interests of two different targets. In this case, the corresponding Polygon of Interests overlaps each other by a small amount as indicated in Figure 10(b). According to our algorithm, first the Overlapped Area of Interest of the two Polygon of Interests, $X$ and $Y$ will be calculated. Let it be named as $Z$. Then the area of $X$ and $Y$ included in $Z$ will be calculated; let them be denoted as $X 1$ and $Y 1$ respectively. We will now calculate $X^{`}$ and $Y^{`}$ using $X^{`}=X$ $-X 1$ and $Y^{`}=Y-Y 1$ respectively. Now we calculate the number of $W N \mathrm{~s}$ in $X^{\prime}$ and $Y^{\prime}$ and denote them as $\mathrm{n}\left(X^{\prime}\right)$ and $n\left(Y^{\prime}\right)$. According to Figure 10, $n\left(X^{\prime}\right)=5$ and $n\left(Y^{\prime}\right)=$ 5 and so the minimization will be preserved according to our algorithm.

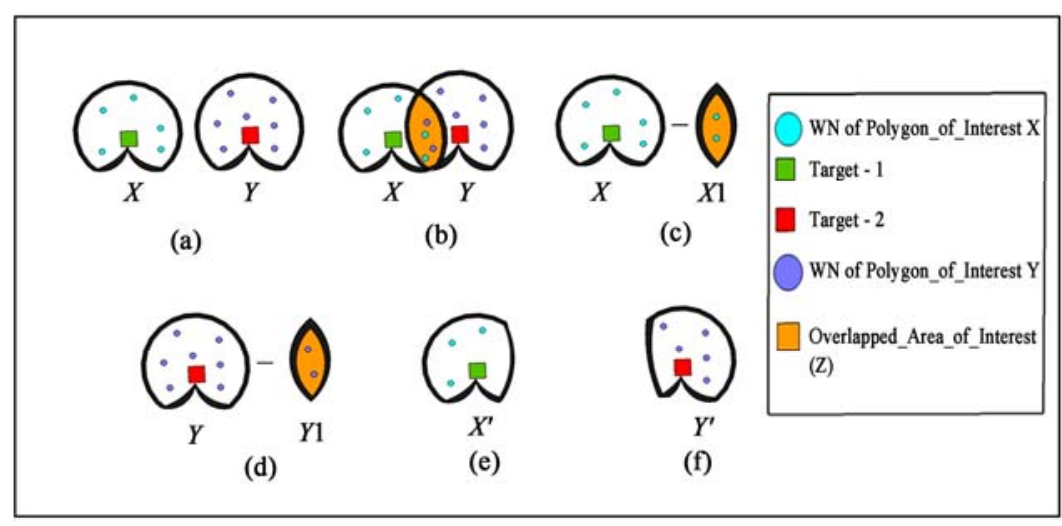

Figure 8. Illustration of CASE 5.

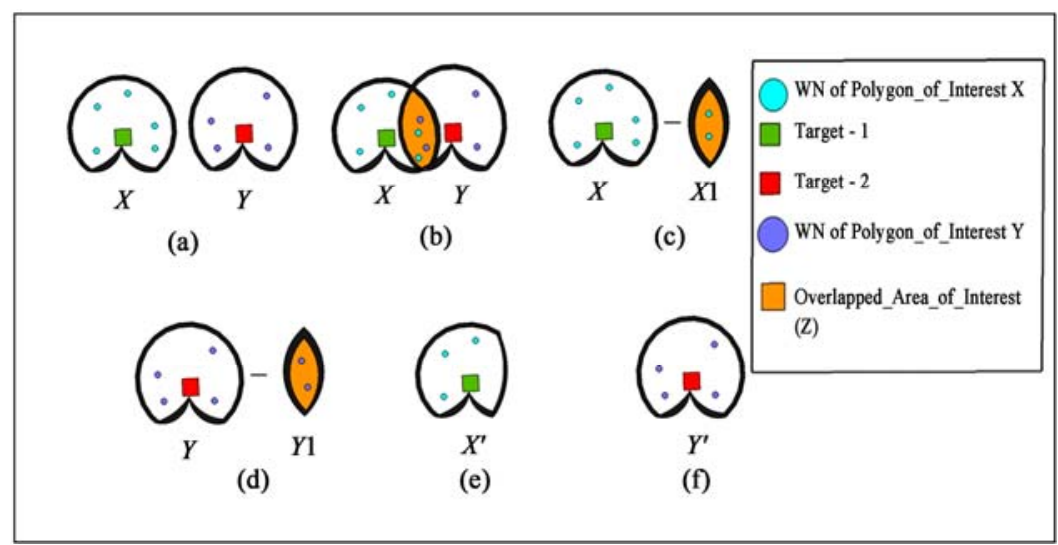

Figure 9. Illustration of CASE 6. 


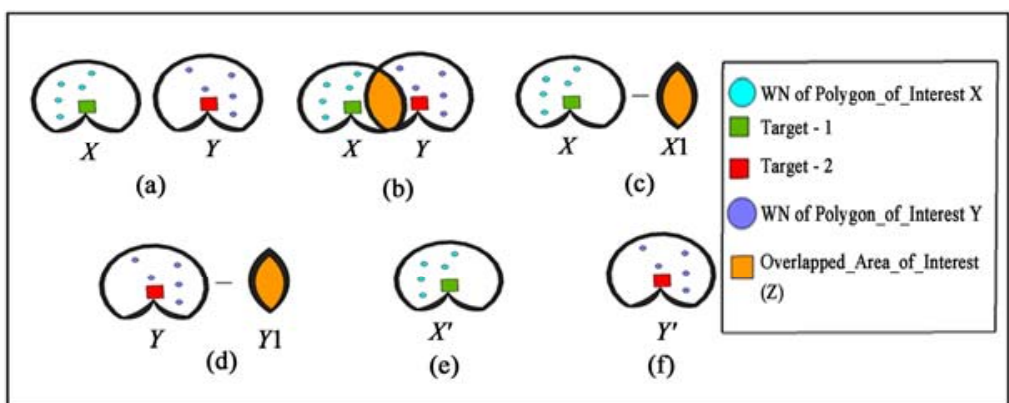

Figure 10. Illustration of CASE 7.

The minimizations for both the Polygon of Interests $X$ and $Y$ have not occurred in terms of number of $W N \mathrm{~s}$ as the Overlapped Area of Interest $Z$ did not contain any $W N \mathrm{~s}$ and hence the number of $W N \mathrm{~s}$ in both the Polygon of Interests remains unchanged.

\section{Evaluation and Comparison}

We simulate using a widely used sensor network simulator OMNeT++ for validating our method. We keep the settings (Table 1) same for all the simulation runs. We set a realistic range for target speed to be $5-12 \mathrm{~ms}^{-1}(18$ - $42 \mathrm{~km}$ per hour). For wheeled vehicles, the steering angle is $20^{\circ}$ in most of the cases [17]. A greater steering angle at such speed is not realistic, and if it is considered, the vehicle is expected to loop within a circular path well inside the Polygon of Interest. The size of the Polygon of Interest is dependent on the refresh time. Appropriate algorithms [17] are used to determine an optimal refresh time. To observe the performance of our tracking algorithm, we simulate using two/three targets moving across the sensor network at the same time, in all the simulation runs. In our first experiment, we implement such a tracking methodology where circular tracking area is used.

We call it 'Centralized Scheme'. We name our second experiment as 'Centralized Scheme with Target Kinematics'. In this experiment we implement MCTA [5]. In the final experiment, we implement our tracking algorithm, MTTP. In accordance to MTTP, we partition the whole tracking field into cells, giving each $C N$ authority over the $W N \mathrm{~s}$ in the relevant cell. Figure 11, 12 and $\mathbf{1 3}$ respectively; show the amount of energy consumed by the $C N \mathrm{~s}, W N \mathrm{~s}$ and the total network. Clearly, the first approach that used circular tracking area consumes more energy at each phase of the simulation. In Table 2, the comparison among three methods is given. We find that our method MTTP outperforms the circular tracking area based algorithms as it prunes out the tracking area approximately by $50 \%$ while preserving up to $60 \%$ of total energy.
Table 1. Simulation Settings.

\begin{tabular}{|c|c|}
\hline Parameter & Value \\
\hline \multicolumn{2}{|c|}{ General Settings } \\
\hline Network Dimension & $1000 \times 1000$ meter \\
\hline Number of $W N \mathrm{~s}$ & 500 \\
\hline Number of $C N \mathrm{~s}$ & 1 to 10 \\
\hline Number of $B N \mathrm{~s}$ & 40 \\
\hline Deployment of nodes & Uniform Random \\
\hline Link types & $\begin{array}{l}\text { 1. } B N \text { to } C N \text { unidirectional, short range } \\
\text { 2. } W N \text { to } C N \text { unidirectional, short range } \\
\text { 3. } C N \text { to } C N \text { unidirectional, long range }\end{array}$ \\
\hline \multicolumn{2}{|c|}{ Event Settings } \\
\hline Simulation type & Discrete-event driven \\
\hline Target intrusion point & User defined \\
\hline Inter-arrival time for targets & Static \\
\hline Target Mobility Type & Brownian motion \\
\hline Simulation Time & $2500 S T U$ \\
\hline \multicolumn{2}{|c|}{ Energy Model } \\
\hline Initial Energy in $W N \mathrm{~s}$ & $100 \mathrm{~J}$ \\
\hline Initial Energy in $B N \mathrm{~s}$ & $100 \mathrm{~J}$ \\
\hline Initial Energy in $C N \mathrm{~s}$ & $2000 \mathrm{~J}$ \\
\hline $\begin{array}{l}\text { Power dissipation in radio } \\
\text { transmission }\end{array}$ & $10^{-4} \mathrm{~W} / \mathrm{m}^{2}$ \\
\hline$W N$ energy dissipation rate & $\begin{array}{l}\text { Active Mode: } 24 \mathrm{~mW} \\
\text { Idle Mode: } 45 \mu \mathrm{W}\end{array}$ \\
\hline$C N$ energy dissipation rate & $\begin{array}{l}\text { Active Mode: } 24 \mathrm{~mW} \\
\text { Idle Mode: } 45 \mu \mathrm{W}\end{array}$ \\
\hline
\end{tabular}

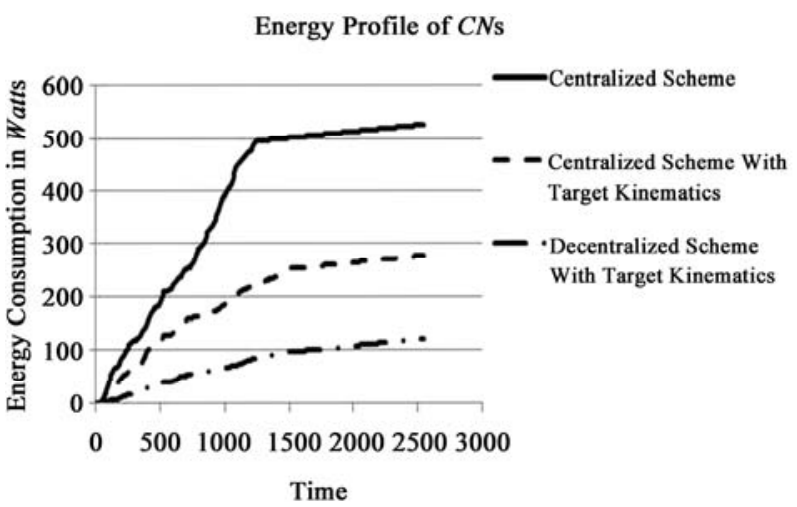

Figure 11. Comparison of energy consumption at CNs. 


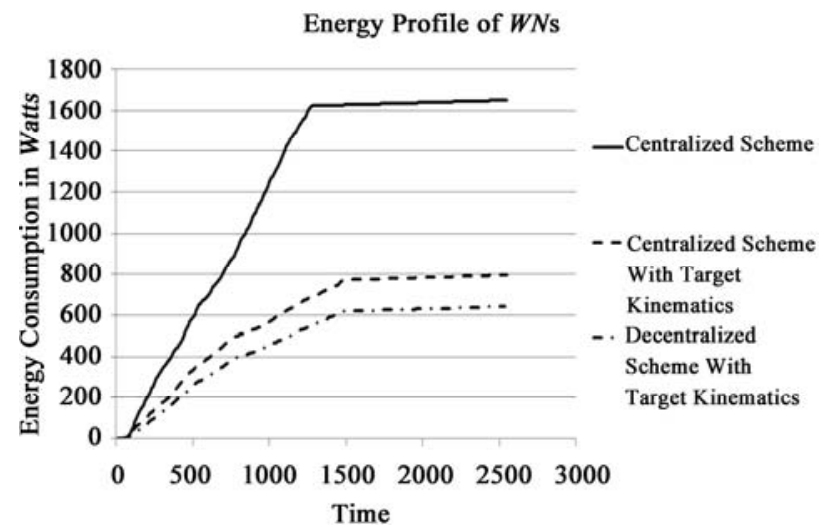

Figure 12. Comparison of energy consumption at WNs.

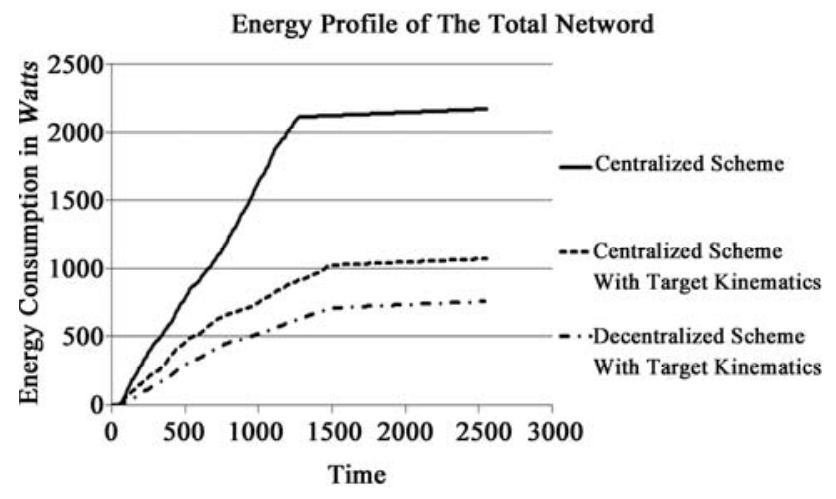

Figure 13. Comparison of energy consumption in the total network.

Table 2. Simulation results.

\begin{tabular}{lccc}
\hline \multicolumn{1}{c}{ Simulation Name } & Centralized Scheme & $\begin{array}{c}\text { Centralized Scheme with } \\
\text { Target Kinematics }\end{array}$ & $\begin{array}{c}\text { Decentralized Scheme with } \\
\text { Target Kinematics }\end{array}$ \\
\hline Tracking Method & Centralized & Centralized & $\begin{array}{c}\text { Distributed } \\
\text { Polygon of Interest }\end{array}$ \\
Tracking Area & Circular & 2500 STU & 2500 STU \\
Simulation Time & 2500 STU & $1525 \mathrm{STU}$ & 1525 STU \\
Tracking Time & $1525 \mathrm{STU}$ & 136 & 136 \\
Total Polygon of Interests formed & 136 & 41 & 41 \\
Polygon of Interests Overlapped & 53 & 10.38 & 10.38 \\
Average WNs per Polygon of Interest & 19.6 & 21 & 21 \\
Maximum WNs per Polygon of Interest & 33 & $795.5 \mathrm{~J}$ & $640.2 \mathrm{~J}$ \\
Total dissipated energy in $W N \mathrm{~s}$ & $1646.5 \mathrm{~J}$ & $276.6 \mathrm{~J}$ & $121.3 \mathrm{~J}$ \\
Total dissipated energy in $C N \mathrm{~s}$ & $523.2 \mathrm{~J}$ & $1072.2 \mathrm{~J}$ & $761.5 \mathrm{~J}$ \\
Total dissipated energy & $2170.7 \mathrm{~J}$ & 2165 & 2681 \\
Number of transmission packets used & 4703 & $280.02 \mathrm{~m}$ & $120.79 \mathrm{~m}$ \\
Average transmission range & $284.52 \mathrm{~m}$ & $696.50 \mathrm{~m}$ & $429.21 \mathrm{~m}$ \\
Maximum transmission range & $670.43 \mathrm{~m}$ & & \\
\hline
\end{tabular}

\section{Conclusions}

In our paper, we propose a distributed energy efficient tracking method for tracking multiple targets in a large scale sensor network. We simulate our algorithm with a sensor network simulator and compare with other popular methods. We find our method significantly saves sensor nodes' energy. In future, we want to implement our algorithm in a real life scenario. We would like to explore multiple layers deployment of boundary sensors nodes which will increase good tracking service but will increase network cost. This trade-off is still an open research problem.

\section{References}

[1] C. Sharp, S. Schaffet, A. Woo, N. Sastri, C. Karlof, S. Sastry and D. Culler, "Design and Implementation of a Sensor Network and Autonomous Interception," Proceedings of the 2nd European Workshop on Wireless
Sensor Networks, Berkeley, 2005.

[2] G. W. Allen, K. Lorincz, M. Welsh, O. Marcillo, J. Johnson, M. Ruiz and J. Lees, "Deploying a Wireless Sensor Network on an Active Volcano," IEEE Internet Computing, Vol. 10, No. 2, March-April 2006, pp. 18-25. doi:10.1109/MIC.2006.26

[3] E. Yoneki and J. Bacon, "A Survey of Wireless Sensor Network Technologies: Research Trends and Middleware's Role," Ph.D. Dissertation, University of Cambridge, Cambridge, 2005.

[4] W. Zhang and G. Cao, "Dynamic Convoy Tree based Collaboration for Target Tracking in Sensor Networks," IEEE Transactions on Wireless Communication, Vol. 3, No. 5, September 2004, pp. 1689-1701. doi:10.1109/TWC.2004.833443

[5] J. Jeong, T. Hwang, T. He and D. Du, “(MCTA) Target Tracking Algorithm based on Minimal Contour in Wireless Sensor Networks," Technical Report, University of Minnesota, Twin Cities, 2007.

[6] S. M. LaValle, "Planning Algorithms," Cambridge University Press, Cambridge, 2006. 
[7] OMNeT++ Community Site. http://www.omnetpp.org

[8] Y. Bar-Shalom and T. E. Fortmann, "Tracking and Data Association," Academic Press Professional, San Diego, 1987.

[9] H. Yang and B. Sikdar, "A Protocol for Tracking Mobile Targets Using Sensor Networks," Proceedings of the IEEE Workshop on Sensor Network Protocols and Applications, Vol. 7, 2003, pp. 71-81. doi:10.1109/SNPA.2003.1203358

[10] Tinynode. http://www.tinynode.com

[11] W. AlSalih, K. Islam, Y. N. Rodriguez and H. Xiao, "Distributed Voronoi Diagram Computation in Wireless Sensor Networks," Proceedings of the 20th ACM Symposium on Parallelism in Algorithms and Architectures (SPAA), 2008, p. 364. doi:10.1145/1378533.1378597

[12] X. Du and F. Lin, "Maintaining Differentaited Coverage in Heterogenous Sensor Networks," EURASIP Journal on Wireless Communication and Networking, Vol. 2005, No. 54, August 2002, pp. 459-461. doi:10.1155/WCN.2005.565
[13] Bob Stein, “A Point About Polygons," Linux Journal, March 1997.

[14] J. A. Stankovic, T. Abdelzaher, and T. He, "Lightweight Detection and Classification for Wireless Sensor Networks in Realistic Environments," Proceedings of the 3rd ACM Conference on Embedded Networked Sensor Systems, San Diego, November 02 - 04 2005, pp. 205-217. doi:10.1145/1098918.1098941

[15] R. R. Brooks, P. Ramanathan and A. M. Sayeed, "Distributed Target Classification and Tracking in Sensor Networks," Proceedings of the IEEE, Vol. 31, No. 8, 2003, pp. 1163-1171. doi:10.1109/JPROC.2003.814923

[16] A. U. R. Akond, M. A. I. Mollah and M. Naznin, "Multiple Targets Tracking in Wireless Sensor Networks using Target Kinematics," Undergraduate Thesis, Bangladesh University of Engineering and Technology, Dhaka, 2009.

[17] D. R. Kincaid and W. W. Cheney, "Numerical Analysis the Mathematics of Scientific Computing," American Mathematical Society, Rhode Island, 2002. 